\title{
A commentary on "Long-term outcome of retropubic tension free vaginal tape for stress urinary incontinence after a trans obturator tape failure; a retrospective study"
}

\author{
Ghazaleh Rostaminia ${ }^{1}$ \\ Received: 29 October 2019 / Accepted: 12 November 2019 / Published online: 11 December 2019 \\ (C) The International Urogynecological Association 2019
}

This retrospective study aimed to investigate the efficacy and safety of a retropubic mid-urethral sling (RP-TVT) procedure after prior trans-obturator sling (TOT) failure for treating stress urinary incontinence. Fifty-one women who presented with persistent or recurrent stress urinary incontinence after a TOT procedure were recruited. All women received a comprehensive assessment including a pelvic examination, supine stress test, cystoscopy in case of irritative urinary symptoms, introital ultrasonography to assess the position of the first sling in case of voiding dysfunction, and a multichannel urodynamic study prior to the second procedure. Follow-up visits after RP-TVT were performed at 1, 6, and 12 months and yearly thereafter.

The median time between the TOT and RP-TVT procedures was 18 months, and the median follow-up after the RP-TVT procedure was 103.2 months $(\mathrm{IQR}=53.8-$ 128.3 months). Postoperative complications occurred in 16 women (39\%) including voiding dysfunction, de novo urinary urgency, and sling erosion. Survival estimates showed that the likelihood of stress urinary incontinence to be cured or improved at $3,5,7$, and 10 years was $78.0 \%, 75.4 \%, 71.9 \%$, and $67.4 \%$, respectively. The absence of urethral hypermobility was the only risk factor for RP-TVT failure $(\mathrm{OR}=11.3$; 95\% CI $=1.2-102.9$ ).

In summary, this study confirms that offering a retropubic TVT procedure to the patients with a failed prior transobturator procedure can improve the symptoms of stress urinary incontinence in approximately $70 \%$ of patients long term, noting that an absence of urethral hypermobility will increase the odds for TVT failure. Failure rates of almost $30 \%$, even after a second mid-urethral sling, highlight our incomplete understanding of urinary continence mechanisms, which warrants further mechanical investigations.
Ghazaleh Rostaminia

ghazalerostaminia@yahoo.com

1 University of Chicago Pritzker School of Medicine, NorthShore University HealthSystem, Skokie, IL, USA 